\section{Is there place for placebo controlled trials in the treatment of affective disorders?}

\author{
Há espaço para ensaios \\ clínicos controlados por \\ placebo no tratamento de \\ transtornos afetivos?
}

The randomised controlled trial (RCT) is the gold standard for measuring quality, safety and efficacy of new medical treatments. In the absence of other effective treatments, the placebo controlled design, which compares a neutral intervention with a purportedly beneficial intervention, is the process by which most novel treatments achieve acceptance by the scientific community. Recently, however, there has been considerable debate about ethical issues surrounding use of placebos in disease areas where effective treatments are already known to exist. For example, placebo-controlled studies expose recipients to an ineffective treatment that may sometimes precipitate adverse consequences. Studies comparing a novel treatment with an already wellestablished approach, by contrast, do not. Many ethical review bodies have therefore become reluctant to sanction use of placebos, preferring as an alternative, trials that use a known active treatment for comparison. Effective pharmacological and psychological approaches have become well established in the treatment of affective disorders, so it is timely to ask whether there are still good methodological and ethical grounds to support the continued use of placebo-controlled studies.

We turn our attention first to methodological issues. It is well known that the number of patients required to discriminate between two interventions increases as the effect size diminishes. In other words, the smaller the difference in benefit between two treatments, the more people you need enrolled in a study to demonstrate that benefit. In active-comparator studies, the measurable benefit of one intervention over a well-established treatment is usually quite small and so these kinds of studies may require very large numbers to demonstrate superiority or even equivalence. While placebo-controlled studies are not completely immune to this problem (for example, placebo response rates can be quite high in the treatment of affective disorders, meaning that treatment effect sizes can be small relative to other disease areas), the general profile is that they require considerably fewer participants than active-comparator studies.

For example, in the treatment of obsessive compulsive disorder (OCD) there has been a striking trend towards increasing placebo response rates over 3 decades (Figure 1). Nonetheless, the sample sizes required to test efficacy against placebo remain favourable compared to those required for comparator-controlled studies. Placebo controlled trials are, therefore, smaller, quicker and cheaper to run. The economic burden of developing new treatments is substantial and so, by reducing impediments to testing, the pharmaceutical industry is more likely to undertake development work with drugs that have novel mechanisms of action. ${ }^{1}$ Trial cost and feasibility are not ethical issues and it is important to keep them separate. However, taking a longer-term perspective, the importance of a commercial incentive in the development of medical treatments cannot be overlooked.

One important advantage of placebo-controlled studies is that, by referencing a new drug to placebo, we can derive clinically relevant constants for the drug such as its effect-size, and the numbers needed to treat. These approaches permit absolute, rather than relative, comparisons with other established treatments.

The methodological advantages of including a placebo-arm need to be offset, however, against ethical issues. The most 


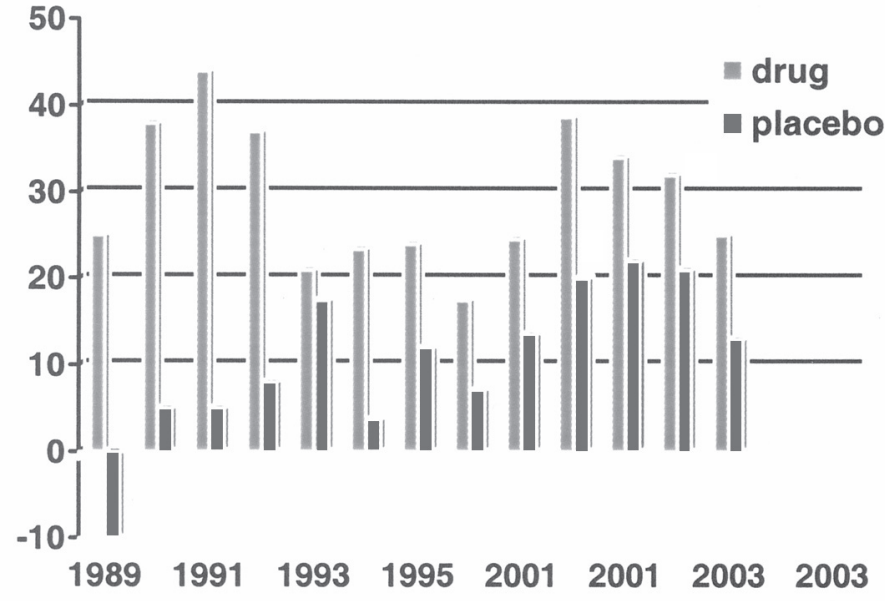

Figure 1 - Mean reduction of baseline Y-BOCS comparing SRI with placebo in OCD trials

difficult criticism to deal with is that patients are necessarily exposed to inefficacious treatments. Of course this does not mean they will not improve; in OCD, for example, placebo-response rates regularly exceed $20 \%$ reduction in baseline severity; but they are unlikely to improve as much on placebo as on an effective treatment. Other harmful consequences of inadequately treated affective disorders include prolonged psychosocial impairment (e.g., job-loss, family breakdown) and even suicidal behaviour. Importantly, patients entering treatment studies are usually screened to exclude those judged to be at risk of suicidality and those with comorbidities may be excluded also.

If we accept the argument that there are methodological and pragmatic arguments for continuing to use placebos in treatment trials, how then should we safeguard patients to ensure they are not subjected to unacceptable risk?

Any placebo-controlled study should be designed to limit numbers exposed, and duration of exposure, to placebo. Moreover, attention to study recruitment criteria is paramount. Patients at high risk of suicidal behaviour or who are unlikely to tolerate the rigours of being in a study should not be included. Informed written consent, explaining the method for randomisation, allocation of treatment and use of placebo, and the patient's non-prejudicial right to withdraw at any time and receive conventional treatment are prerequisite. Patients need to be monitored carefully. This usually means more frequently than for conventional clinical practice, and research staff must be available for consultation between appointments if necessary. Explicit withdrawal criteria are needed either for non-response or adverse effects, and there should be a protocol for following up and treating those individuals who leave the study early, or who remain symptomatic at the end of the trial

In the absence of viable alternatives, placebo-referenced studies offer some convincing advantages over active-controlled trials in acute and long-term studies of affective disorders. Safe use of placebos requires careful planning and novel techniques for limiting exposure to ineffective treatment within the context of treatment trials should be explored. Expert consensus involving clinicians, ethicists, medical statisticians and patients with affective disorders would undoubtedly be welcomed by the medical and scientific community.
Naomi A Fineberg

Post-Graduate Medical School, University of Hertfordshire, College Lane, Hatfield, UK

Departament of Psychology, University of Hertfordshire, Hatfield, UK

Departament of Psychiatry, Queen Elizabeth II Hospital, Hertfordshire Partnership NHS Trust, Welwyn Garden City, UK

Tim M Gale

Departament of Psychology, University of Hertfordshire, Hatfield, UK

Departament of Psychiatry, Queen Elizabeth II Hospital, Hertfordshire Partnership NHS Trust, Welwyn Garden City, UK

Chris J Hawley

Post-Graduate Medical School, University of Hertfordshire, College Lane, Hatfield, UK

Departament of Psychiatry, Queen Elizabeth II Hospital, Hertfordshire Partnership NHS Trust, Welwyn Garden City, UK

\section{Reference}

1. Fritze J, Moller HJ. Design of clinical trials of antidepressants: should a placebo control be included? CNS Drugs. 2001;15(10):755-64. 